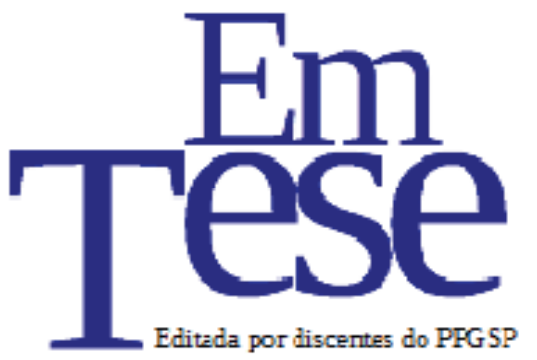

UFSC

PPG SP PROG RAMA DE

PÓS-GRADUAÇÃO EM

Sociologia

Política

\title{
Desigualdade, desenvolvimento humano e corrupção nos estados brasileiros $^{*}$
}

\author{
Willber Nascimento ${ }^{1}$ \\ Anderson Henrique ${ }^{2}$ \\ Antônio Alves Tôrres Fernandes ${ }^{3}$
}

Resumo: Qual a relação entre desenvolvimento humano, desigualdade de renda e corrupção no Brasil? O objetivo do trabalho é testar a hipótese de que corrupção está negativamente correlacionada com o IDHM e positivamente associada a desigualdade de renda nos estados. Metodologicamente, utiliza-se estatística descritiva, correlação de Pearson e regressão linear de múltiplos quadrados ordinários (MQO). Os resultados encontrados indicam que: (1) há uma associação positiva entre desigualdade de renda e corrupção; (2) desenvolvimento humano está correlacionado negativamente com a corrupção e; (3) de maneira geral, quando se aumenta a desigualdade de renda em uma unidade observa-se um aumento de 1,803 no Índice de Corrupção dos Estados Brasileiros (ICE).

Palavras-chave: corrupção; morosidade judicial; unidades da federação.

\section{Inequality, human development and corruption in the Brazilian states}

Abstract: What is the relation between human development, income inequality and corruption in Brazil? The main point of this paper is to test hypothesis that corruption is negatively correlated with human development and positively associated to income inequality in Brazilian states. Methodologically, the research design combines descriptive and multivariate statistics with Pearson correlation and Ordinary Least Square (OLS) models. The main results indicate that: (1) there is a positive association between income inequality and corruption; (2) human development is negatively correlated with corruption and; (3) in general, when income inequality increases in one unit it notices which has a growth in 1,803 in the Brazilian States Corruption Index.

Keyword: corruption; judicial delays; federative units.

\footnotetext{
* Somos gratos a uma série de pessoas pela ajuda na construção deste artigo. Dentre elas mencionamos os pareceristas da Revista em Tese que nos alertou sobre várias coisas importantes que não havíamos pensado. Agrademos aos membros do Grupo de Pesquisa de Métodos de Pesquisa em Ciência Política (UFPE). Destacamos que falhas contidas aqui são de inteira responsabilidade dos autores.

1 Doutorando em Ciência Política pela Universidade Federal de Pernambuco (UFPE). E-mail: willbernascimento@outlook.com.

2 Graduando em Ciência Política pela Universidade Federal de Pernambuco (UFPE). E-mail: andersonheri@gmail.com.

3 Graduando em Ciência Política pela Universidade Federal de Pernambuco (UFPE). E-mail: antonioalvestorres123@ hotmail.com.
} 


\section{Introdução}

A corrupção tem sido um fenômeno complexo para a produção de estudos científicos devido à baixa quantidade de dados disponíveis. Normalmente, os estudos são realizados com base em dados de percepção e não de dados observacionais. Isso porque, tanto os gestores quanto agências do Estado tendem a não possuir ou mesmo disponibilizar informações. Muitos dos indicadores elaborados para medir a corrupção são construídos a partir da percepção de empresários, gestores e experts que indicam através de surveys o grau de corrupção de um determinado país ou instituição (GEHLBACH, 2009).

Dentre os principais órgãos internacionais que disponibilizam dados baseados em surveys sobre corrupção, destacam-se o Banco Mundial e a Transparência Internacional. Concomitantemente, no Brasil temos o Cadastro Nacional de Improbidade Administrativa disponibilizado pelo Conselho Nacional de Justiça (CNJ) que atualiza casos de improbidade por parte de agentes públicos julgados pelos tribunais de esferas estaduais e federais ${ }^{4}$, o Tribunal de Contas da União (TCU) com as análises das contas municipais ${ }^{5}$, o Ministério Público Federal (MPF) com o ranking da Transparência ${ }^{6}$, entre outros. Buscando expandir essa área de estudo no Brasil, analisamos a relação entre corrupção, o desenvolvimento humano e a desigualdade de renda por unidades da federação.

Este trabalho está dividido em quatro seções, além da introdução e considerações finais. Na primeira, apresentamos uma breve revisão bibliográfica acerca da corrupção, desenvolvimento humano e desigualdade de renda. Na segunda seção, discutimos as dificuldades em mensurar a corrupção e apresentamos alguns indicadores disponíveis no Brasil para acessá-la. Na terceira seção, apresentamos a metodologia aplicada na elaboração e análise dos dados. Em seguida, na quarta seção, analisaremos os dados e testaremos a hipótese de trabalho.

\footnotetext{
${ }^{4}$ O Conselho Nacional de Justiça tem disponibilizado através do seu site informações sobre os casos julgados por improbidade administrativa nas três esferas do governo (municipal, estadual e federal). Por sua vez, esses casos são classificados por diferentes áreas. Alguma informação relevante é disponível, como a pena aplicada, o valor da multa e informações sobre perda de cargo/mandado por parte do agente público. Para mais informações:

http://www.cnj.jus.br/improbidade_adm/consultar_requerido.php. Acessado em 21 de maio de 2015.

${ }^{5}$ Para mais informações:

http://portal.tcu.gov.br/responsabilizacao-publica/contas-julgadas-irregulares/.

${ }^{6}$ Disponível em: http://rankingdatransparencia.mpf.mp.br/.
} 


\section{Breves apontamentos sobre desigualdade, desenvolvimento humano e corrupção}

Corrupção é uma agenda de pesquisa que tem recebido muita atenção nos últimos anos. Diversas áreas de estudos vêm tentando lidar com as diversas problemáticas colocadas pela dinâmica do objeto "corrupção". A Economia e a Ciência Política são áreas que dedicam enorme esforço para estudar esse fenômeno, tentando estimar possíveis causas e efeitos. Buscamos apresentar um panorama acerca desses estudos, focalizando, principalmente, a relação entre desigualdade social e corrupção.

Iniciemos por uma definição. Existem diversas formas de conceituar, além do que existem vários ramos da corrupção. Por isso, ter uma única definição não é uma tarefa simples. Majoritariamente, no entanto, os pesquisadores da área têm formulado uma definição estrita acerca do fenômeno da corrupção. Desse modo, para muitos desses pesquisadores, entende-se a corrupção como o abuso do poder público, ou cargo público, para ganhos e fins privados (YOU e KHAGRAM, 2005; TREISMAN, 2000). É bem verdade que existe uma outra gama de estudos que analisa a corrupção social de maneira mais geral. Dentre esses estudos destacam-se aqueles sociológicos, antropológicos e psicológicos (GACHTER e SCHULZ, 2016).

Estudar corrupção, portanto, não é uma tarefa simples. Isso porque, a maior parte dos atos corruptos tendem a ser mantidos em segredo e, em sua grande maioria, não são descobertos. Logo, a disponibilidade de dados é reduzida. Tentando superar esse problema, pesquisadores de maneira geral têm estudado corrupção basicamente a partir de dados de percepção. Segundo Gehlbach (2009), estudos cross-national sobre corrupção exploram dados oriundos de avaliação de experts e elaboração de surveys por empresas, institutos de pesquisa, ONGs, entre outros ${ }^{7}$. Nesse sentido, as pesquisas sobre corrupção têm um perfil muito similar: (1) comparam países (cross-national); (2) utilizam dados de percepção; e (3) analisam quais são suas causas e os efeitos.

Por outro lado, as causas da corrupção é uma questão frequente nesse tipo de estudo. Segundo You e Khagram (2005), elas podem ser dividas em variáveis de ordem econômica, política, histórica e cultural. As variáveis econômicas mais convencionais são: desenvolvimento econômico, extensão e níveis de educação, tamanho da classe

\footnotetext{
${ }^{7}$ Avaliação de experts diz respeito a uma série de perguntas que especialistas respondem sobre os níveis de corrupção no país em que eles vivem. Enquanto que os surveys são questionários que, normalmente, cidadãos (amostra) de um país respondem acerca dos níveis de corrupção em seu país.
} 
média, abertura de mercado, recursos naturais, entre outros. Do lado das variáveis políticas encontramos basicamente regime político, tamanho do governo e descentralização política, econômica e funcional (YOU e KHAGRAM, 2005). Por fim, as variáveis históricas e culturais mais frequentes são: religião, valores morais, herança colonial e fracionalização linguística e cultural ${ }^{8}$.

Os principais estudos utilizam o desenvolvimento econômico como uma das principais causas da corrupção. Os resultados desses estudos apontam para uma forte relação entre desenvolvimento econômico e corrupção. Pesquisadores têm observado que quanto maior for o desenvolvimento econômico do país menores serão os seus indicadores de corrupção (YOU e KHAGRAM, 2005; TREISMAN, 2000; FIGUEIREDO FILHO, 2013).

Apesar disso pouca atenção tem sido dada a relação entre desigualdade social e corrupção (YOU e KHAGRAM, 2005; BECOVIG, 2006). You e Khagram (2005) argumentam que quanto mais desigual for uma sociedade maior serão seus indicadores de corrupção. Becovig (2006), por sua vez, afirma que existem dois tipos principais de desenho de pesquisa acerca dessa relação. O primeiro focaliza o efeito da corrupção sobre a mudança da desigualdade econômica, à medida que o segundo, analisa a desigualdade econômica enquanto um fator explicativo da corrução e vice-versa (BECOVIG, 2006).

No primeiro caso, argumenta-se que a corrupção exerce um efeito significativo sobre a distribuição da desigualdade. Em termos mais específicos, a corrupção tende a manter e, até mesmo, difundir desigualdades principalmente na distribuição de renda ${ }^{9}$. Por outro lado, assume-se que quanto mais desigualdade maior será a corrupção. Ou seja, a existência da alta desigualdade aumenta o espaço para as ações de corrupção ${ }^{10}$.

Grande parte desses estudos esperam uma relação positiva entre corrupção e desigualdade social. Segundo You e Khagram (2005), desigualdade de renda aumenta a corrupção porque ela a promove para um patamar de comportamento aceitável. Quando

\footnotetext{
${ }^{8}$ Para mais informações acerca das causas da corrupção ver Ades e Di Tella (1999), Paldam (2002), Treisman (2000), Montinola e Jackman (2002), La Palombara (1994), La Porta et al (1999), Paldam (2001), Gachter e Shulz (2016).

9 Alguns mecanismos apontados por Gupta et al (2002) são: (1) sistema de tributação enviesado; (2) orientação não eficaz dos programas sociais devido à corrupção está "sugando" os recursos; (3) gastos para a formação educacional reduzidos graças à corrupção; (4) aumenta incertezas e riscos para os pobres. ${ }^{10}$ Para maior profundidade no argumento ver Alam (1997), Scott (1972), Husted (1999), Hellmanand Kaufman (2002), Uslaner (2005), You e Khagram (2005), Alesina e Angeletos (2005).
} 
uma sociedade é extremamente desigual tanto ricos, quanto pobres tendem a enxergar as práticas corruptas como aceitáveis. Isso porque onde os pobres são privados de serviços sociais básicos tais como saúde, educação, moradia e segurança, há uma maior probabilidade de entregar-se a prática corrupta rotineiramente. Ainda, segundo os autores, essas práticas estão relacionadas à tentativa de suborno à burocracia, dentre elas instâncias como na administração de escolas, cartórios, hospitais, polícia, entre outros. Como os pobres alcançam esses pequenos benefícios, legitimam suas práticas corruptas e também as práticas dos ricos (normalmente associadas a "necessidade" de manter suas posições).

Em estudo recente Gachter e Shulz (2016) identificaram que em países com alta prevalência de violação das regras a honestidade das pessoas tende a ser afetada. Nesse caso, onde impera a violação das regras os indivíduos tendem a aceitar práticas de corrupção sem ter sua alto-imagem de honestidade posta em risco. Nesse caso, práticas de corrupção passam a ser toleradas.

Por outro lado, a relação entre desigualdade social e corrupção é vista como uma via de mão dupla. A corrupção também pode reforçar as desigualdades existentes (YOU e KHAGRAM, 2005). Ela cria um círculo vicioso onde desigualdade gera corrupção que mantém ou aumenta as desigualdades já existentes. Portanto, a desigualdade afeta a maneira como a corrupção é percebida entre todos os membros de determinada sociedade. You e Khagram (2005) concluem que a desigualdade de renda pode ser uma variável tão importante quanto o desenvolvimento econômico uma vez que se deseja estudar a corrupção. Destaca-se que a desigualdade econômica não é a única forma de desigualdade. Do mesmo modo, o desenvolvimento humano pode dizer bastante sobre a desigualdade social visto que compreende três indicadores (renda, educação e longevidade).

\section{Missão quase impossível: mensurando corrupção no Brasil}

Como mencionado acima, estudar corrupção é algo extremamente complicado. Devido à natureza do ato corrupto, a disponibilidade de dados observacionais é reduzida. Desse modo, mensurar a corrupção é um dos grandes desafios quando se pretende estudá-la. Alguns estudos nacionais tentam mensurar a corrupção e são 
descritos nessa seção. Abaixo apresentamos e descrevemos o indicador de morosidade judicial em casos de improbidade administrativa e corrupção, o Índice de Corrupção Governamental Estadual (ICE) e o ranking da transparência.

Em primeiro lugar destacamos o indicador de morosidade judicial como proxy para a corrupção proposto por Figueiredo Filho et al (2013). Esse indicador se baseia na média do tempo para se julgar um caso de improbidade administrativa e ou corrupção. Em relação às ações por improbidade administrativa, o tempo de julgamento ainda é maior do que em relação aos outros crimes. Figueiredo Filho et al (2013) argumentam que a ação de improbidade têm por finalidade dois objetivos: (1) punir os ímprobos por seus atos ao dano erário ou uso indevido do cargo em prol de si e/ou terceiros e (2) reaver os valores dos prejuízos causados ao Estado indevidamente.

Mello (2010) define a improbidade por parte do agente público (direto ou indiretamente) pela falta de honestidade e adversidades por parte dos atores às normas da moral e da lei, assim como dos bons costumes. A Constituição Federal brasileira de 1988, por exemplo, no seu art. 37 estabelece que a administração pública (direta ou indireta) terá que seguir princípios que mantenham a lisura da impessoalidade, eficiência, legalidade, publicidade e moralidade, em que os infratores julgados poderão receber penas que variam desde a perda do mandato político e cargo de natureza pública e/ou ressarcimento ao Estado através de multas e ações penais civis cabíveis. Apesar destas especificações na Constituição Federal (CF) 88, a Lei Nº 8.429/92 aparece como um reforço contra os atos de improbidade administrativa na administração pública direta, indireta ou fundacional ${ }^{11}$.

A impunidade pode ser pensada como um mecanismo que liga morosidade a corrupção. Uma vez que os Tribunais se tornam omissos à corrupção dado o tempo que levam para julgar estes casos, principalmente quando envolve políticos, a sociedade passa a não atribuir credibilidade e eficiência a estas instituições. Além disso, passa-se a impressão de que os tribunais não punem os políticos. $\mathrm{O}$ gráfico 1 compara o tempo médio de julgamento de casos de corrupção com outros tipos de processo na esfera

\footnotetext{
${ }^{11}$ Para mais informações sobre a Lei 8.429/92 ver: <http://www.planalto.gov.br/ccivil_03/leis/18429.htm>. Acessado em 19 de maio de 2015.
} 
estadual com base nos dados disponibilizados pelo Conselho Nacional de Justiça entre 1992 e $2015^{12}$.

Gráfico 1-Morosidade Judicial estadual por tipo de processo (IC 95\%)

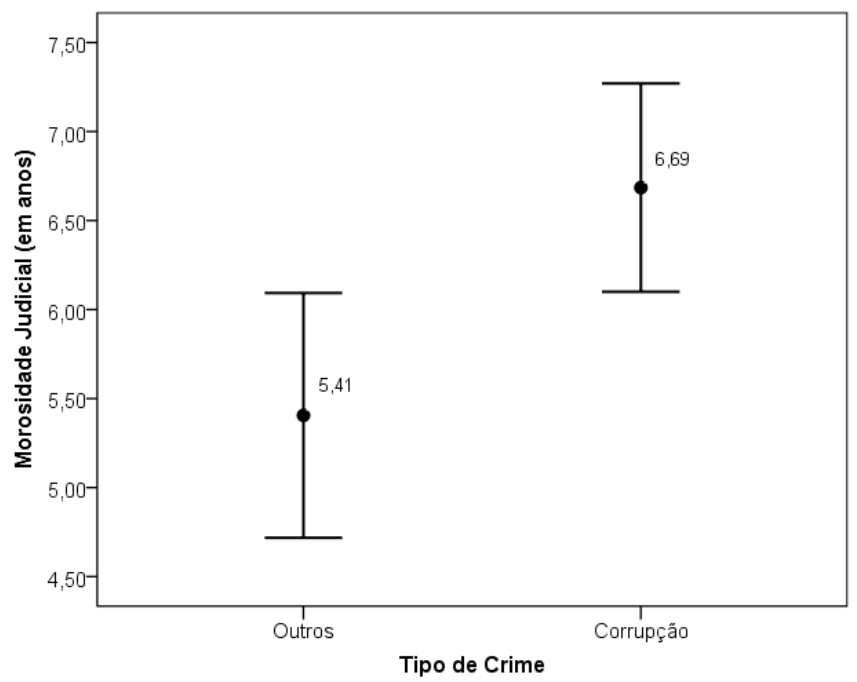

Fonte: Elaboração dos autores (2016).

Os tribunais estaduais demoram, em média, 1,28 ano a mais para julgar casos de corrupção quando comparado com os outros tipos de processo. Essa diferença é estatisticamente significativa ( $\mathrm{p}$-valor $=0,005$ ). Do mesmo modo, é possível perceber esse efeito nos casos julgados em esfera federal. O gráfico 2 apresenta essa comparação.

\footnotetext{
${ }^{12}$ Esses dados foram coletados diretamente na página do CNJ, por meio de uma ferramenta de coleta automática de dados. Para mais informações sobre o procedimento acessar: https://www.academia.edu/24446142/TOGARY_a_program_for_automated_data_collection.
} 


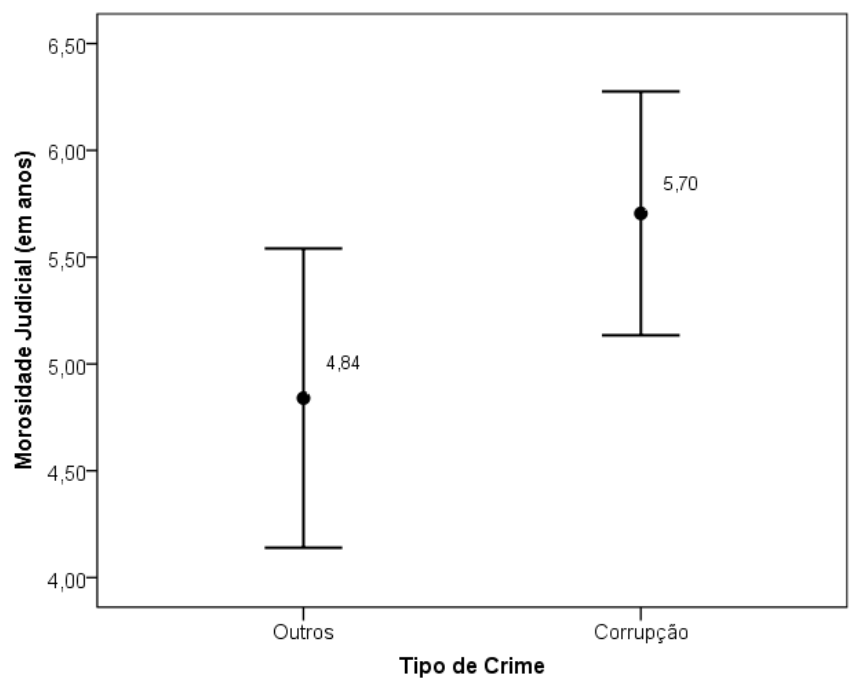

Fonte: Elaboração dos autores (2016).

No caso dos tribunais federais, processos de corrupção são julgados com uma média de 0,86 ano a mais para julgar casos de corrupção quando comparado com os outros tipos de processo. Essa diferença é estatisticamente significativa (p-valor = $0,029)^{13}$.

Estudos recentes têm apontado que a honestidade das pessoas diminui em sociedade que possuem um alto nível de violação da lei (GACHTER e SCHULZ, 2016). Um judiciário, portanto, que não julga em tempo hábil e às vezes não pune adequadamente atos de corrupção, contribui para a corrupção ${ }^{14}$. Isto é, espera-se que em estados onde o judiciário demora para julgar e punir envolvidos em corrupção e improbidade administrativa exista um maior nível de corrupção.

Outro indicador de corrupção em nível estadual é o Índice de Corrupção Governamental Estadual (ICE) desenvolvido por Boll (2010). Ele possui intuito de mensurar a corrupção nos estados brasileiros de modo a contornar o problema de

\footnotetext{
${ }^{13}$ Por outro lado, em estudo de revisão de literatura sobre fluxo do sistema judiciário brasileiro em perspectiva comparada, Ribeiro e Silva (2010) tem identificado que a taxa de resolução dos crimes é baixa em diversos ramos da justiça, além disso, que a justiça tende a demorar para julgar casos em todos os tipos de crimes. Estes dados divergem daqueles que o CNJ disponibiliza. Essas divergências, mais do que tudo, apontam para a necessidade de futuros estudos sobre esse tema e da abertura transparente dos órgãos de justiça do Brasil que, em sua maioria, podem ser vistos como "caixas pretas" quando comparado com demais instituições do Estado brasileiro.

${ }^{14}$ Por exemplo, Santos (2014) identificou que os tribunais estaduais passaram a julgar com menor severidade os casos de corrupção e improbidade administrativa no Brasil.
} 
subjetividade presente em outros indicadores, tais como o Índice de Corrupção da Transparência Internacional que utiliza dados de percepção. Foram utilizados na construção do indicador dados do Tribunal de Contas da União referentes ao resultados financeiros das contas julgadas irregulares encontradas no CADIRREG (Cadastro de Responsáveis de Contas Julgadas Irregulares) ${ }^{15}$, valores executados das despesas correntes e de capital, sendo excluídos os valores referentes às transferências de capital e os recursos específicos destinados às regiões do país obtidos na Lei Orçamentária Anual (LOA), dados populacionais em relação a cada unidade da federação obtidos no IBGE e, por fim, os dados referentes ao Produto Interno Bruto (PIB) de cada estado que foram obtidos no site do Instituto de Pesquisa Econômica Aplicada (IPEA). Para a construção do ICG foram estabelecidos, primeiramente, indicadores simples. Essas informações estão sumarizadas no quadro 1 apresentado abaixo.

\section{Quadro 1 - Indicadores simples}

\begin{tabular}{|l|l|}
\hline \multicolumn{1}{|c|}{ Variável } & \multicolumn{1}{|c|}{ Descrição } \\
\hline Valor CADIRREG/População & $\begin{array}{l}\text { O componente indica o valor das irregularidades per } \\
\text { capita para cada estado. }\end{array}$ \\
\hline Valor CADIRREG/PIB & $\begin{array}{l}\text { O componente indica o valor das irregularidades com } \\
\text { relação ao PIB de cada estado. }\end{array}$ \\
\hline Valor CADIRREG/LOA & $\begin{array}{l}\text { O componente indica o valor das irregularidades com } \\
\text { relação ao volume de recursos da LOA aplicados em } \\
\text { cada estado. }\end{array}$ \\
\hline $\begin{array}{l}\text { Número anual de processos irregulares } \\
\text { registrados no CADIRREG por estados / } \\
\text { número total anual de processos irregulares } \\
\text { registrados no CADIRREG por estados } \\
(1998 / 2008)\end{array}$ & $\begin{array}{l}\text { O componente indica o percentual correspondente ao } \\
\text { estados. }\end{array}$ \\
\hline
\end{tabular}

Fonte: Elaboração dos autores (2016), com base em Boll (2010).

Para a construção do índice de corrupção governamental estadual (ICE), o autor utilizou as seguintes ponderações: peso de 0,33 às variáveis socioeconômicas (população e PIB estadual) e 0,66 em relação a LOA e a quantidade de processos irregulares (BOLL, 2010). Uma grande vantagem desse índice é a de que ele lida diretamente com casos de corrupção em âmbito municipal apontados por uma agência

\footnotetext{
15 Para mais informações, acessar o portal: http://portal.tcu.gov.br/responsabilizacao-publica/contasjulgadas-irregulares/. Acessado em 05 de julho de 2016.
} 
de controle do Estado brasileiro. O gráfico 3 apresenta a distribuição do ICE para os estados da federação.

\section{Gráfico 3 - Média do índice de corrupção governamental estadual}

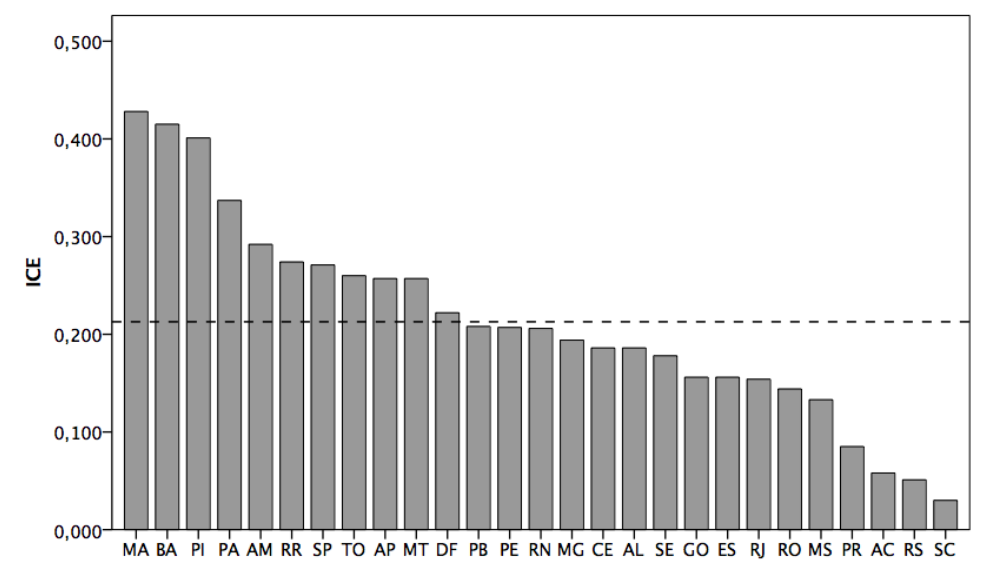

Fonte: Elaboração dos autores (2016), com base em Boll (2010).

O estado que apresenta o maior índice de corrupção governamental é o Maranhão com um valor de 0,428 , enquanto que Santa Catarina é o estado com o menor valor no índice de corrupção governamental com 0,03. A média dos valores é de 0,213.

Outra medida que pode ser utilizada como um proxy de corrupção advém de uma iniciativa do Ministério Público Federal (MPF) em criar um indicador de Transparência em nível estadual e municipal ${ }^{16}$. A transparência de modo geral e, em particular, das contas públicas é algo fundamental para uma democracia. A própria $\mathrm{CF}$ de 1988, no capítulo VII art. 37, coloca como um dos princípios básicos da administração pública direta e indireta de qualquer dos Poderes da União, dos Estados, do Distrito Federal e dos Municípios obedecerá a publicidade. A transparência pode ser considerada um antônimo de corrupção, já que este tem como característica básica atos realizados na surdina. Com o passar do tempo, novos instrumentos legais contribuíram para o fortalecimento desse princípio. Exemplos que fortaleceram a transparência na administração pública foram: a Lei Complementar $\mathrm{n}^{\circ} 101$ do ano 2000 (lei de responsabilidade fiscal), a Lei Complementar $n^{\circ} 131$ de 2009 e a Lei de Acesso à informação (Lei n $12.257 / 11)$.

\footnotetext{
${ }^{16}$ Para mais informações, acessar o portal: http://rankingdatransparencia.mpf.mp.br/. Acessado em 08 de julho de 2016.
} 
O ranking da transparência faz parte de uma série de medidas tomadas pelo MPF no combate à corrupção. O propósito do ranking é verificar se os estados e munícipios brasileiros estão cumprindo as normas relacionadas à transparência e controle dos gastos públicos. O MPF adotou uma série de medidas para os munícipios que não estavam cumprindo as leis da transparência como, por exemplo, a recomendação para que a União suspenda os repasses de transferências voluntárias. Os itens avaliados ${ }^{17}$ pelo MPF na construção do ranking da transparência foram coletados por meio de um questionário elaborado em conjunto com outras instituições responsáveis pelo controle e fiscalização como a Controladoria Geral da União (CGU), Tribunal de Contas da União (TCU), Banco Central (BC), entre outros.

A figura abaixo apresenta o ranking da transparência estadual e municipal. Em termos operacionais o ranking da transparência municipal será apresentado agregadamente pelos estados. Em termos técnicos, ele é a média da transparência dos municípios de um dado estado. O ranking da transparência estadual é relacionado ao nível de transparência do governo do estado.

Figura 1 - Ranking da Transparência Municipal e Estadual

(A) Ranking da transparência municipal (2015)

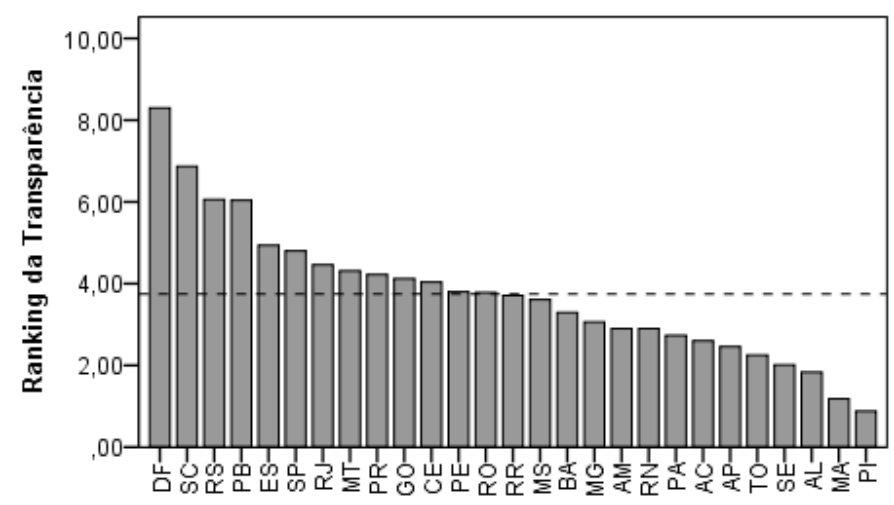

(B) Ranking da transparência municipal (2016)

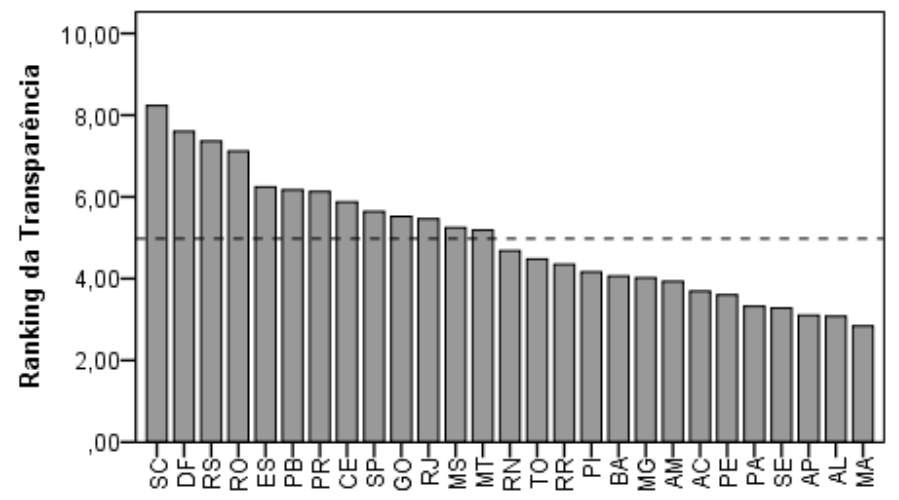

17 Para mais informações acerca dos itens avaliados ver:

$<$ http://combateacorrupcao.mpf.mp.br/ranking/o-projeto-new/ranking/itens-avaliados $>$. Acessado em 6 de julho de 2016. 
(C) Ranking da transparência Estadual (2015)

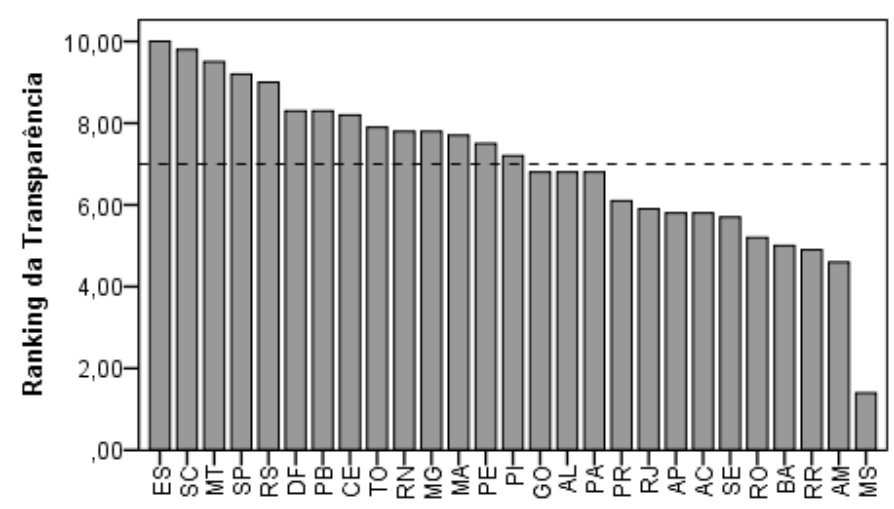

(D) Ranking da transparência Estadual (2016)

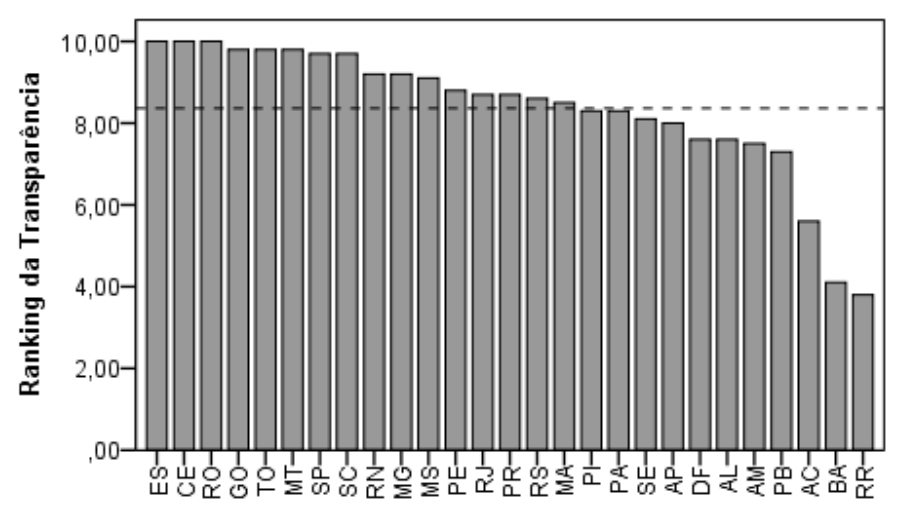

Fonte: Elaborada pelos autores (2016).

A linha pontilhada indica a média da distribuição. No caso da transparência municipal (A-B) os estados do Distrito Federal e de Santa Catarina são aqueles em que os municípios são mais transparentes. E a distribuição possui maior variância. No caso da Transparência do Governo Estadual, Espirito Santo, Santa Catarina e Ceará são mais transparentes. Abaixo apresentamos o nível de correlação entre estes três indicadores para cada período de tempo de que dispomos de dados. A tabela 1 sumariza esses resultados.

Tabela 1 - Correlação de Pearson entre os indicadores de corrupção

\begin{tabular}{|c|c|c|c|c|c|c|}
\hline Variáveis & ICE & $\begin{array}{c}\text { Morosidade } \\
\text { Judicial }\end{array}$ & $\begin{array}{c}\text { Transparência } \\
\text { Municipal } \\
2015 \\
\end{array}$ & $\begin{array}{c}\text { Transparência } \\
\text { Municipal } \\
2016 \\
\end{array}$ & $\begin{array}{c}\text { Transparência } \\
\text { Estadual } \\
2015 \\
\end{array}$ & $\begin{array}{c}\text { Transparência } \\
\text { Estadual } \\
2016\end{array}$ \\
\hline ICE & 1 & 0,005 & $-0,483 * *$ & $-0,559 * *$ & $-0,075$ & $-0,295$ \\
\hline Morosidade Judicial & & 1 & $-0,162$ & $-0,240$ & $-0,013$ & $-0,383^{*}$ \\
\hline Transparência Municipal 2015 & & & 1 & $0,857 * *$ & $0,394 *$ & 0,128 \\
\hline Transparência Municipal 2016 & & & & 1 & $0,363 *$ & $0,353 *$ \\
\hline Transparência Estadual 2015 & & & & & 1 & $0,424 *$ \\
\hline
\end{tabular}

Transparência Estadual 2016

$\mathrm{N}=27$; Teste com uma extremidade; ** A correlação é significativa no nível 0,01 (1 extremidade); * A correlação é significativa no nível 0,05 (1 extremidade).

Fonte: Elaboração dos autores (2016).

As correlações entre ICE e transparência estadual não são significativas, mas caminham na direção esperada. Ou seja, governos estaduais menos transparentes tendem a apresentar um maior ICE. Contudo, o ICE não está relacionado a morosidade 
judicial. Por outro lado, o ICE apresenta uma correlação negativa de magnitude moderada e significativa com a transparência municipal de ambos os anos analisados. Substantivamente, espera-se que um aumento no ICE produza uma diminuição na transparência municipal. No que se refere a morosidade judicial, ela apresenta o padrão de correlação negativa esperado (estados com tribunais que demoram mais para julgar possuem menor transparência), mas só apresenta um resultado com maior magnitude e significância com a transparência estadual de $2016^{18}$.

Utilizaremos para as análises propostas nesse artigo o ICE. O Índice de Corrupção Governamental Estadual apresenta as seguintes vantagens: (1) O recorte temporal do ICE é maior; (2) O ICE é calculado com base nos casos de corrupção e improbidade administrativa indicados pelo TCU; (3) O ICE apresenta um padrão de correlação consistente com as expectativas teóricas com os demais indicadores disponíveis. A próxima seção apresenta a metodologia que subsidia a análise dos dados.

\section{Metodologia}

Nesta seção apresentamos os principais aspectos metodológicos do trabalho com o objetivo de maximizar a transparência da confecção e análise dos dados. Nesse trabalho lidamos com dados observacionais sobre corrupção e comparamos pelos estados. Como salientou Guelbach (2009), dados agregados tendem acobertar padrões de corrupção diferentes entre as unidades geográficas de um mesmo país. Como medida de corrupção utilizamos o Índice de Corrupção Governamental Estadual (ICE) desenvolvido por Boll (2010). O quadro abaixo resume as informações acerca dessa variável.

\section{Quadro 2 - Variável dependente}

\begin{tabular}{|c|l|l|}
\hline Variável dependente & \multicolumn{1}{|c|}{ Descrição } & \multicolumn{1}{c|}{ Período e Unidade de Análise } \\
\hline Corrupção & $\begin{array}{l}\text { Média do Índice de Corrupção } \\
\text { Governamental Estadual (ICE). }\end{array}$ & $\begin{array}{l}\text { Dados referentes ao período de } 1998 \text { até } \\
2008 .\end{array}$ \\
\hline
\end{tabular}

Fonte: Elaboração dos autores (2016).

\footnotetext{
18 A importância do P-valor é reduzida devido à baixa quantidade de casos (27). O foco da análise repousa sobre a direção das correlações.
} 
Operacionalmente, a corrupção é medida de acordo com os procedimentos indicados por Boll (2010) discutidos acima. O quadro 3 sumariza as variáveis independentes do trabalho.

Quadro 3 - Variáveis independentes

\begin{tabular}{|c|l|c|}
\hline $\begin{array}{c}\text { Variável } \\
\text { Independente }\end{array}$ & \multicolumn{1}{|c|}{ Operacionalização } & $\begin{array}{c}\text { Período e Unidade } \\
\text { de Análise }\end{array}$ \\
\hline GINI & $\begin{array}{l}\text { Índice de desigualdade de renda que varia de 0 a } \\
\text { 1.Onde, quando mais próximo 1 mais desigual é a } \\
\text { região. }\end{array}$ & 1991 a 2010 \\
\hline IDHM & $\begin{array}{l}\text { Índice de desenvolvimento humano que varia de 0 a } \\
\text { 1. Em que quanto mais próximo de 1 mais } \\
\text { desenvolvido é a região. }\end{array}$ & 1991 a 2010 \\
\hline PIB per capita & Média do PIB per capita. & 1991,2000 e 2010. \\
\hline
\end{tabular}

Fonte: Elaborada pelos autores (2016).

As variáveis independentes GINI e IDHM foram coletadas no site do Atlas Brasil $^{19}$. Por outro lado, nossa variável de controle, o PIB per capta, foi coletado no site do IBGE $^{20}$ e no relatório do IPECE ${ }^{21}$. De modo geral, nossas variáveis independentes medem a desigualdade econômica e social nos Estados. Os estudos analisados utilizam o Gini como medida de desigualdade. Além dela, usamos o IDHM como uma variável agregada de qualidade de vida. Baseados na literatura utilizada testaremos as seguintes hipóteses:

$\mathrm{H}_{1}$ : GINI está positivamente associado a corrupção.

$\mathrm{H}_{2}$ : IDHM está associado negativamente a corrupção.

$\mathrm{Na}$ próxima seção apresentamos e analisamos os dados de corrupção, desigualdade social e qualidade de vida nos estados brasileiros.

\footnotetext{
${ }^{19}$ Disponível em: <http://seculoxx.ibge.gov.br/economicas/contas-nacionais>. Acessado em 08 de julho de 2016.

${ }^{20}$ Disponível em:

$<$ http://www.ipece.ce.gov.br/publicacoes/ipece-informe/Ipece_Informe_46_28_novembro_2012.pdf $>$. Acessado em 08 de julho de 2016.

${ }^{21}$ Disponível em <http://www.atlasbrasil.org.br/2013/pt/consulta/>. Acessado em 08 de julho 2016.
} 


\section{Resultados}

Esta seção apresenta os principais resultados do trabalho. Nosso objetivo é analisar a distribuição da média do ICE (1998-2008) no Brasil e sua relação com a desigualdade social e o desenvolvimento humano. Nossa premissa teórica é a de que o nível de desigualdade em uma dada sociedade afeta os níveis de corrupção observados. A tabela 2 apresenta a distribuição do ICE no Brasil.

Tabela 2 - Estatística descritivos do ICE no Brasil

\begin{tabular}{c|c|c|c|c}
\hline $\mathrm{N}$ & Mínimo & Máximo & Média & Desvio Padrão \\
\hline 27 & 0,03 & 0,428 & 0,213 & 0,104 \\
\hline
\end{tabular}

Fonte: Elaboração dos autores (2016).

A média da distribuição da corrupção no Brasil é de 0,213 , com um desvio padrão de 0,104. O Estado que apresenta a menor média de corrupção é Santa Catarina com 0,03 , enquanto o Maranhão é aquele com maior média de corrupção $(0,428)$. Para uma visualização a figura 2 apresenta a distribuição do ICE no mapa do Brasil.

Figura 2- Mapa da média do Índice de Corrupção Governamental Estadual (ICE)

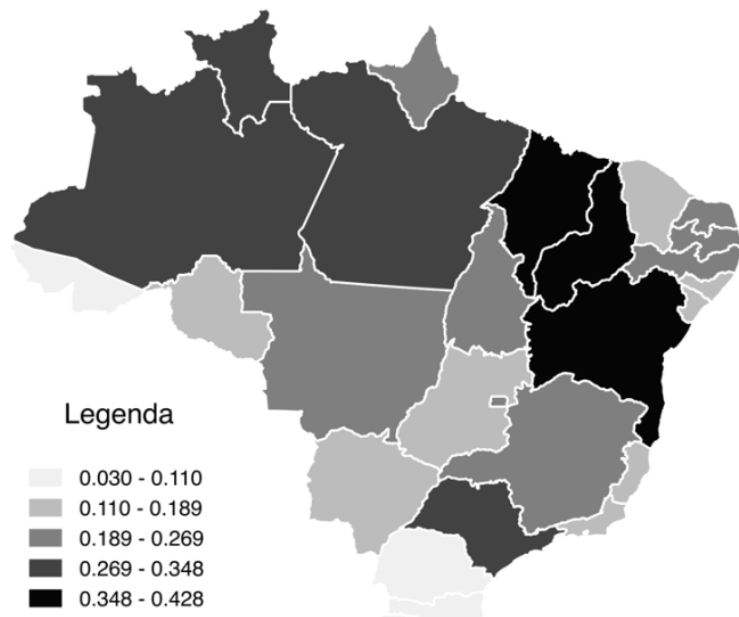

Fonte: Elaboração dos autores (2016). 
Exceto São Paulo, o centro-oeste e o sul são as regiões do país que aparecem com o menor ICE quando comparado com as outras regiões. Sergipe, Alagoas e Ceará são os estados do nordeste com menor incidência de corrupção de acordo com o ICE. Por outro lado, também são da região nordeste os estados com maior ICE: Bahia $(0,415)$, Maranhão $(0,428)$ e Piauí $(0,401)$.

Agora avançaremos diretamente para a análise das expectativas teóricas. Iniciaremos por apresentar a relação entre a desigualdade de renda nos estados e o índice de corrupção ICE. O gráfico abaixo sumariza essas informações com os dados disponíveis do Gini.

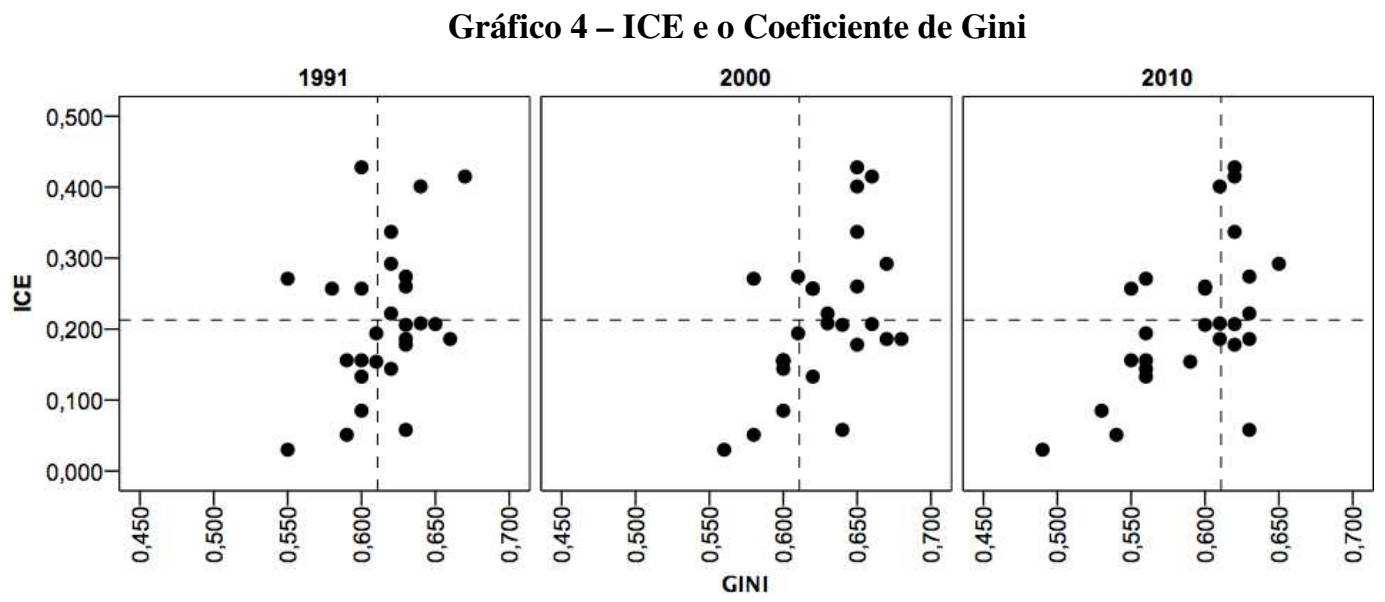

Fonte: Elaboração dos autores (2016).

Observa-se uma nítida relação positiva entre o Gini e a corrupção no estado. Em termos descritivos, encontramos empiricamente a direção esperada da relação estados com mais desigualdade de renda apresentam índices de corrupção mais elevados. Adicionalmente analisamos a magnitude dessas correlações. A tabela 3 apresenta um teste de correlação de Gini e corrupção.

Tabela 3 - Correlação do ICE e do Coeficiente de Gini

\begin{tabular}{|c|c|c|c|c|}
\hline Variáveis & ICE & GINI 1991 & GINI 2000 & GINI 2010 \\
\hline ICE & 1 & $0,335^{*}$ & $0,524 * *$ & $0,556 * *$ \\
\hline GINI 1991 & & 1 & $0,775^{* *}$ & $0,694 * *$ \\
\hline GINI 2000 & & & 1 & $0,830 * *$ \\
\hline GINI 2010 & & & & 1 \\
\hline
\end{tabular}


De acordo com os coeficientes de correlação, existe uma relação moderada entre desigualdade de renda e os níveis de corrupção nos estados analisados. Adicionalmente, essa relação apresenta-se significativa ao nível de p-valor $<0,01$. Em relação ao Gini de 1991, o coeficiente de correlação é de 0,335, um patamar abaixo dos posteriores. Salientamos que neste estamos correlacionando o Gini da década de 81-91 com a corrupção média entre 1998 e 2010. De certo modo, essa relação indica uma dependência explicativa no tempo indicando que a corrupção em um instante $t+1$ pode estar relacionada a desigualdade no instante t. $\mathrm{O}$ gráfico 5 apresenta a relação entre o IDHM e corrupção.

\section{Gráfico 5 - ICE e o IDHM}

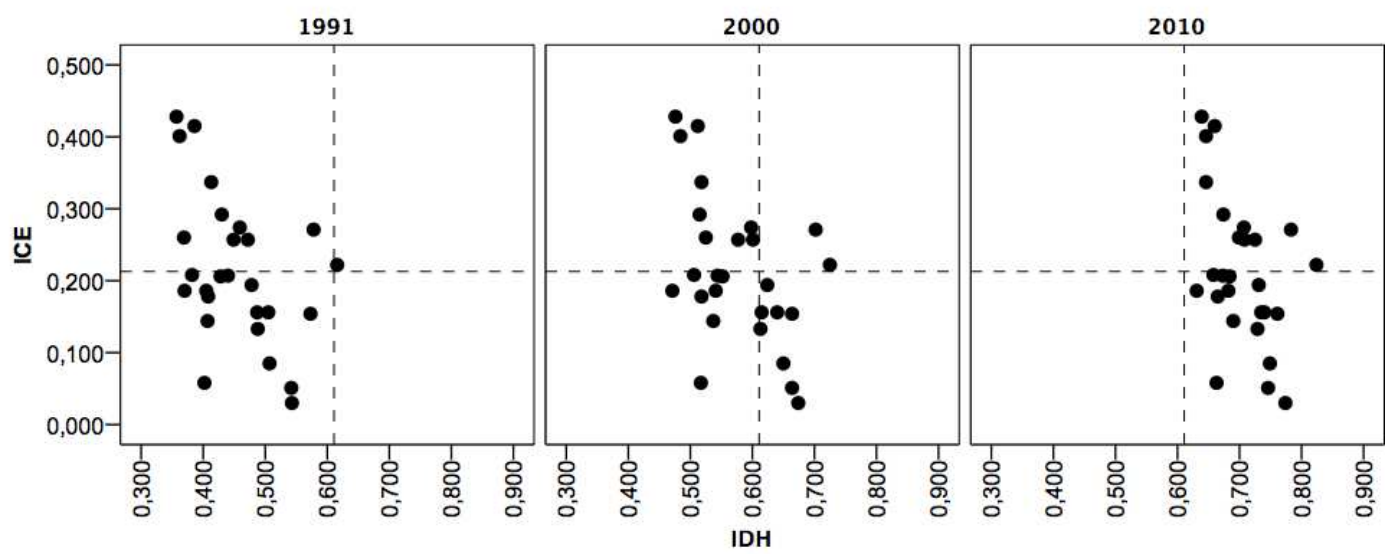

Fonte: Elaboração dos autores (2016).

A relação entre IDHM e ICE é claramente negativa. Mais uma vez, esses resultados condizem com os apontamentos teóricos. Estados onde o desenvolvimento humano é melhor possuem menor nível de corrupção. A força dessa relação é apresentada abaixo. A tabela 4 apresenta os coeficientes de correlação de Pearson.

Tabela 4 - Correlação entre o ICE e o IDHM

\begin{tabular}{ccccc}
\hline Variáveis & ICE & IDHM 1991 & IDHM 2000 & IDHM 2010 \\
\hline ICE & 1 & $-0,455^{* *}$ & $-0,472 * *$ & $-0,449 * *$ \\
IDHM 1991 & 1 & $0,971^{* *}$ & $0,943 * *$ \\
IDHM 2000 & & 1 & $0,979 * *$ \\
IDHM 2010 & & 1 \\
\hline N = 27; Teste com uma extremidade; \\
** A correlação é significativa no nível 0,01; \\
* A correlação é significativa no nível 0,05. \\
Fonte: Elaboração dos autores (2016).
\end{tabular}


Os resultados encontrados na tabela 4 apontam que existe uma relação negativa de magnitude moderada e significativa ( $\mathrm{p}$-valor < 0,005) entre o ICE e o IDHM. O nível de associação do IDHM 1991 com o ICG é de -0,455, enquanto que com o IDHM de 2000 o nível de associação é de -0,472. Com o IDH de 2010, o nível de associação é de $-0,449$. Os valores encontrados apontam que a medida que desenvolvimento humano aumenta observa-se uma diminuição na corrupção governamental. Mas qual o efeito do desenvolvimento humano e da distribuição de renda na corrupção dos estados brasileiros? Abaixo faremos esse exercício.

Com base nas expectativas teóricas apresentadas ao longo do artigo esperamos um efeito positivo do GINI sobre a corrupção e um negativo do IDHM sobre a corrupção. Utilizamos um modelo de regressão de mínimos quadrados ordinários $(\mathrm{MQO})^{22}$ para fazer essas análises. Podemos pensar o modelo da seguinte maneira:

$$
Y=\alpha+\beta 1_{x 1}+\beta 2_{x 2}+\beta 3_{x 3}+\varepsilon
$$

Onde Y é a variável dependente do modelo, $\alpha$ é a constante, os $\beta$ são as variáveis independentes e $\varepsilon$ representa o termo de erro do modelo. No caso dos nossos objetivos podemos expressar esse modelo como o esboçado na equação abaixo:

$$
Y(I C E)=\alpha+\beta 1_{\text {Gini }}+\beta 2_{\text {Idhm }}+\beta 3_{\text {PIB per }}+\varepsilon
$$

Queremos estimar o tamanho do efeito da desigualdade de renda e posteriormente do desenvolvimento humano sobre a corrupção. Inserimos no nosso modelo a renda per capta para controlar as relações. Salientamos que o número de casos do nosso trabalho (27) é baixo e que, portanto, as significâncias dos testes serão afetadas. A tabela 5 apresenta o modelo de MQO para as médias do Gini, IDH e renda per capita.

\footnotetext{
${ }^{22}$ Para uma introdução ao modelo de regressão de mínimos quadrados ordinários ver Figueiredo Filho $e t$ al (2011).
} 
Tabela 5 - Análise de regressão (MQO) ${ }^{23}$

\begin{tabular}{cccccc}
\hline \multirow{2}{*}{ Variáveis } & \multicolumn{5}{c}{ Modelo } \\
\cline { 2 - 5 } & $1,803^{* *}$ & $1,716^{*}$ & $(3)$ & $(4)$ & $(5)$ \\
\hline \multirow{2}{*}{ Média Gini } & $(0,587)$ & $(0,647)$ & & & 0,812 \\
& & & $-0,757^{*}$ & $-1,522^{*}$ & $-0,969$ \\
Média do IDHM & & $(0,288)$ & $(0,547)$ & $(0,913)$ \\
PIB per capta & &,- 133 & & 1,117 & 0,706 \\
em R $\$ 1.000$ & & $(0,384)$ & & $(0,686)$ & $(0,878)$ \\
\hline Constante & $-88,847 *$ & $-82,496$ & $65,043 * *$ & $100,313^{*} *$ & 22,017 \\
$\mathrm{R}^{2}$ & 0,274 & 0,278 & 0,216 & 0,294 & 0,312 \\
\hline$* * \mathrm{P}<0,01 ; *$ & $\mathrm{P}<0,05 ; \mathrm{N}=27$. ICE, Gini e IDHM variam entre $0-100$. &
\end{tabular}

Fonte: Elaboração dos autores (2016).

No modelo 1 observamos o efeito do Gini sobre o ICE. De acordo com ele, o aumento em uma unidade no Gini aumenta o ICE em 1,803. Quando controlado pelo média do PIB per capta (modelo 2) o efeito diminui para 1,716 indicando certa robustez. No que se refere ao IDHM, seu efeito é negativo (modelo 3). Quando se aumenta o IDHM em uma unidade espera-se uma redução de 0,757 no ICE. Quando controlado pelo PIB per capta (modelo 4) esse efeito passa para -1,522. No modelo 5, todas as variáveis são inseridas na análise. Mantendo-se o IDHM e o PIB per capita constante o efeito do Gini sobre o ICE é de 0,812. Em termos práticos, essa tabela pode indicar uma previsão do ICE de acordo com os níveis de qualquer variável independente. Por exemplo, é esperado que um estado com Gini, IDH e PIB per capita na média possua um nível de corrupção de 19,90 de acordo com o ICE nas estimativas do modelo 5.

\section{Considerações finais}

O objetivo desse trabalho foi observar a relação entre corrupção, desenvolvimento humano e desigualdade de renda no Brasil. Propomos analisar o Índice de Corrupção Governamental dos Estados (ICE) como um indicador de corrupção e compará-lo entre os estados do Brasil, partindo da posição teórica de que

\footnotetext{
${ }^{23}$ Nossa intenção é estimar a direção e o tamanho do efeito das nossas variáveis independentes sobre a corrupção nos estados. Salientamos que este modelo possui algumas limitações: (1) o número de casos é baixo e isso afeta a significância dos testes; (2) IDH e PIB per capta estão muito relacionados, de modo que o tamanho e significância dos coeficientes não são medidos adequadamente pelo modelo de MQO; (3) não inserimos outras variáveis que ajudam a explicar o grau de corrupção do estado. Isso pode ser percebido pelo percentual de variação explicada $\left(\mathrm{R}^{2}\right)$.
} 
quanto maior for a desigualdade de renda e quanto menor for o desenvolvimento humano maior serão os indicadores de corrupção.

A utilização do Índice de Corrupção Governamental Estadual apresenta vantagens como: (1) o recorte temporal do ICE é maior; (2) O ICE é calculado com base nos casos de corrupção e improbidade administrativa indicados pelo TCU; (3) o ICE apresenta um padrão de correlação consistente com as expectativas teóricas. Além disso oferece como principal diferencial contornar o problema de subjetividade presente em outros indicadores usados na literatura especializada.

Os dados analisados sugerem que a desigualdade de renda tem uma associação positiva com o ICE e, em contrapartida, uma associação negativa com desenvolvimento humano. Estes resultados estão em consonância com os resultados dos estudos especializados sobre corrupção. No entanto, diferente desses estudos utilizamos dados observacionais e comparáveis entre subunidades geográficas de um mesmo país através do ICE. Em resumo, os principais resultados encontrados apontam que: (1) há uma associação positiva entre desigualdade de renda e corrupção; (2) há uma associação negativa entre o índice de desenvolvimento humano e corrupção; (3) esses resultados mantêm-se mesmo quando controlados pelo PIB per capita.

Por outro lado, o trabalho possui algumas limitações que podem ser superadas em futuros estudos. Em primeiro lugar, precisamos expandir a série de tempo dos indicadores de corrupção e aprimorá-los para outras esferas. O ICE (BOLL, 2010) é medido basicamente com as infrações a lei registradas em contas municipais selecionadas pelo TCU deixando de fora uma série de outras variáveis importantes. Além disso, é uma medida de corrupção institucional, nada sabemos das práticas de corrupção em nível social. Em segundo lugar, não possuímos uma quantidade de casos razoável para modelos econométricos robustos. Essas análises poderiam ser feitas por municípios para resolver esse tipo de problema.

\section{Referências}

ADES, A.; DI TELlA, R. Rents, Competition and Corruption. American Economic Review, 89:982-94, 1999. 
ALAM, M.S. A Theory of Limits on Corruption and some Applications, Kyklos, Vol. 48, pp. 419-435, 1997.

ALESINA, A.; ANGELETOS, G.M. Corruption, Inequality and Fairness, Journal of Monetary Economics, Vol. 52, pp. 1227-1244, 2005.

BEGOVIC, B. Economic Inequality and Corruption. Working Paper, Center for Liberal-Democratic Studies, Belgrade, Serbia. Disponível em: http://danica.popovic.ekof.bg.ac.rs/206.pdf 2006.

BOLL, J. L. S. A corrupção governamental no Brasil: construção de indicadores e análise da sua incidência relativa nos estados brasileiros. Tese. Pontifícia Universidade Católica do Rio Grande do Sul (PUC-RS), 2010.

SCOTT, J. Comparative Political Corruption, Englewood Cliffs, NJ: Prentice-Hall, 1972.

FIGUEIREDO FILHO, D. B. Justiça tarda, mas não falha? Improbidade Administrativa nos Estados Brasileiros. Working paper, 2013.

; NUNES, F.; ROCHA, E. C.; SANTOS, M. L.; SILVA, M. B.; SILVA JÚNIOR, J. A. O que fazer e o que não fazer com a regressão: pressupostos e aplicações do modelo linear de Mínimos Quadrados Ordinários (MQO). Politica Hoje (UFPE. Impresso), v. 20, p. 44-99, 2011.

GEHLBACH, S. What Can Firm and Household Surveys Tell Us About Expert Assessments of Corruption? Paper presented at the American Political Science Association, Oct., 2009.

GACHTER, S.; SCHULZ, J. F. Intrinsic honesty and the prevalence of rule violations across societies. Nature, 2016.

GUPTA, S.; DAVOODI, H. R.; ALONSO, R. Does Corruption Affect Income Inequality and Poverty? Economics of Governance 3:23-45, 2002.

HELLMAN, J.; KAUFMAN, D. The Inequality of Influence, mimeo, Washington, D.C.: World Bank, 2002.

HUSTED, B. W. Wealth, Culture, and Corruption. Journal of International Business Studies 30:339-60, 1999.

LA PALOMBARA, J. The Structural aspects of corruption. Corruption and Democracy, 1994. 
LA PALOMBARA, J. The Structural aspects of corruption. Corruption and Democracy, 1994.

LA PORTA, Rafael et al. The quality of government. Journal of Law, Economics, and organization, v. 15, n. 1, p. 222-279, 1999.

MELLO, C. A. B. Curso de direito administrativo. 27. ed. São Paulo: Malheiros, 2010. MONTINOLA, Gabriella R.; JACKMAN, Robert W. Sources of corruption: A crosscountry study. British Journal of Political Science, v. 32, n. 01, p. 147-170, 2002.

PALDAM, Martin. Corruption and religion adding to the economic model. Kyklos, v. 54, n. 2-3, p. 383-413, 2001.

PALDAM M. The cross-country pattern of corruption: Economics, culture and the seesaw dynamics, European Journal of Political Economy, 18(2), pp. 215-40, 2002.

PAZZAGLINI, M. F.; ROSA, M. F.; FAZZIO JR., W. Improbidade Administrativa. 3. ed. São Paulo: Atlas, 1998.

RANKING DE TRANSPARÊNCIA DO MINISTÉRIO PÚBLICO FEDERAL. Disponível em: <http://combateacorrupcao.mpf.mp.br/ranking>. Acessado em 6 de julho de 2016.

RIBEIRO, L.; SILVA, K. Fluxo do Sistema de Justiça Criminal Brasileiro:Um balanço da literatura. Cadernos de Segurança Pública. Ano 2, Número 1, Agosto de 2010.

SANTOS, D. S. Improbidade administrativa no Brasil: uma análise de output. Tese. Universidade Federal de Alagoas (UFAL), 2014.

TREISMAN, D. The Causes of Corruption: A Cross National Study. Journal of Public Economics, 76:399-457, 2000.

USLANER, E. M. The Bulging Pockets and the Rule of Law: Corruption, Inequality, and Trust, Paper presented at the Conference: The Quality of Government: What It is, How to Get it, Why It Matters? November 17-19, Goeteborg University, Department of Political Science, 2005.

YOU, J. S.; KHAGRAM, S. A. Comparative Study of Inequality and Corruption. American Journal Association, Vol. 70, No. 1, Feb, 2005, pp. 136-157, 2005.

Recebido em 1 de abril de 2016.

Aprovado em 15 de julho de 2016. 